\title{
B-hadrons \& Heavy Quarkonium: Spectroscopy \& Properties
}

\author{
E. Asli Yetkin $1,3, a$ \\ ${ }^{1}$ on behalf of ATLAS, CMS, and LHCb Collaborations \\ ${ }^{2}$ University of lowa, USA
}

\begin{abstract}
The recent results from the LHC experiments (ATLAS, CMS, and LHCb) are presented in the field of B hadrons and the heavy quarkonium with focus on the properties and the spectroscopy. The observation of the structure in the $J / \psi \phi$ mass spectrum, the $\Lambda_{b}$ lifetime measurements and the production of the $\mathrm{X}(3872)$ via decays to $J / \psi \pi^{+} \pi^{-}$by CMS experiment will be presented for the first time. Additionally the $B_{s}$ lifetime difference measurement, observation of $\chi_{b}(3 P)$ state, observation of the excited $\Lambda_{b}^{0}$ baryons, and the observation of a new baryon $\Xi_{b}^{*}$ will be summarized.
\end{abstract}

\section{Introduction}

Based on the 2011 LHC pp collision data collected at 7 $\mathrm{TeV}$ center of mass energy, the LHC experiments measured the properties and spectroscopy of the B-hadrons and heavy quarkonium sector. The provided measurements are useful tools for providing an insight into the nature of QCD. The LHC experiments are performing analysis in order to observe the structures/resonances that are suggested by the various theoretical model and also increase the measurement precision for the known particle properties. The results of these analysis which are performed by ATLAS, CMS, and LHCb collaborations will be summarized in Sec. 2 for heavy quarkonium field and in Sec. 3 for the B-hadrons field.

\section{Heavy Quarkonium}

\subsection{Observation of the structures in the $J / \psi \phi$ mass spectrum}

The observation of new states such as $X(3872), Y(3940)$, and $Y(4260)[1,5]$, which do not fit into the conventional quark model, renewed the interest in the exotic states. Especially the observation of $Y(3940)$ near the $J / \psi \omega$ threshold motivated similar structure searches near the $J / \psi \phi$ threshold. In 2009, the CDF collaboration studied the $J / \psi \phi$ mass structure in $B^{+} \rightarrow J / \psi \phi K^{+}$decay channel and obtained evidence for a narrow structure, called as $\mathrm{Y}(4140)$, near the $J / \psi \phi$ threshold [6]. In 2011, CDF updated their results with a larger data sample and measured the mass and the width of the observed structure as $4143.4_{-3.0}^{+2.9}$ (stat.) \pm 1.2 (syst.) $\mathrm{MeV}$ and $15.3_{-6.1}^{10.4}$ (stat) \pm 2.5 (sys.) $\mathrm{MeV}$ [7]. Recently, the LHCb collaboration studied the same mass spectrum using 0.37 $f b^{-1}$ of pp collision data produced at $\sqrt{s}=7 \mathrm{TeV}$ at

\footnotetext{
ae-mail: eaa@cern.ch
}

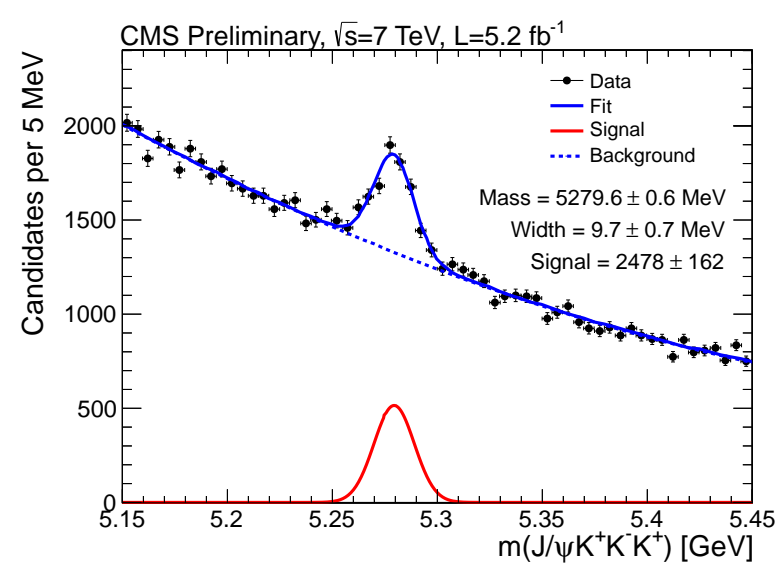

Figure 1. The invariant mass of $J / \psi K^{+} K^{-} K^{+}$after all the event selections. The solid blue line is a fit to the data with a Gaussian signal function and a second order Chebyshev polynomial background function.

the LHC, however they did not confirm the existence of $Y(4140)$ and put an upper limit on its production [8]. With the conflicting reports from the two experiments, a result from an independent experiment is important to resolve the issue.

In order to solve the puzzle, CMS performed an analysis using $5.2 \mathrm{fb}^{-1}$ pp collision LHC data with $7 \mathrm{TeV}$ center of mass energy. The $\mathrm{B}$ candidates are reconstructed in the decay channel $B^{+} \rightarrow J / \psi \phi K^{+}$, where $J / \psi \rightarrow \mu^{+} \mu^{-}$and $\phi \rightarrow K^{+} K^{-}$. The $m\left(J / \psi K^{+} K^{-} K^{+}\right)$spectrum is shown in Fig 1 for the selected B candidates. With $2478 \pm 162$ B candidates, this is the largest $\mathrm{B}$ sample in the world up to date. The invariant mass distribution of the $K^{+} K^{-}$pairs from $J / \psi K^{+} K^{-} K^{+}$candidates within the $3 \sigma$ of the B mass, after sideband subtraction is shown in Fig. 2. The spectrum is fitted with a P-wave relativistic Breit-Wigner function con- 


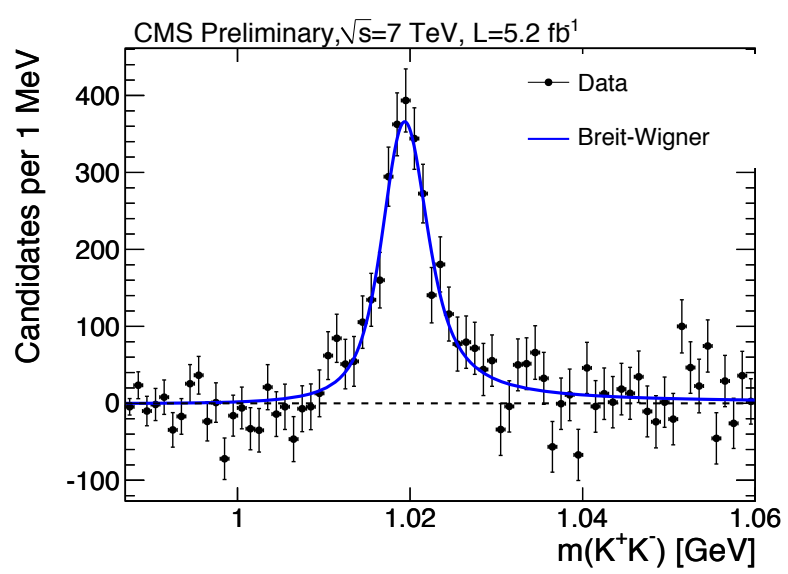

Figure 2. The $B^{+}$sideband subtracted mass distribution of $K^{+} K^{-}$ for $0.987-1.060 \mathrm{GeV}$ region. The distribution is shown before the $\phi$ mass window requirement. The blue solid curve shows the Pwave relativistic Breit-Wigner fit to the data.

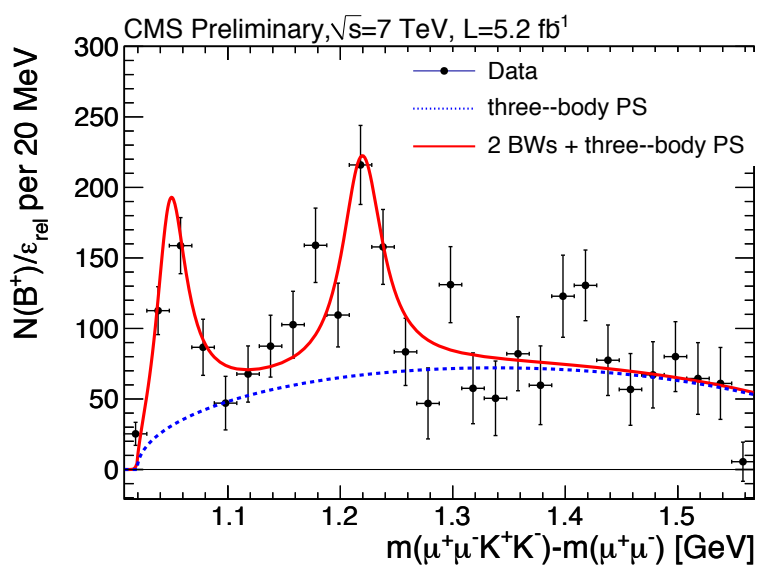

Figure 3. The efficiency corrected $\Delta m$ spectrum with two signalhypothesis fit.

voluted with a Gaussian resolution function. The extracted mass and the width values are consistent with the world average values for the $\phi$ meson [9], which indicates that after the $\phi$ mass window selection the $B^{+} \rightarrow J / \psi K^{+} K^{-} K^{+}$final state well describes the $J / \psi \phi K^{+}$system. For the selected B candidates, the dataset is divided into the $20 \mathrm{MeV} \Delta m$ ( $\equiv m\left(\mu^{+} \mu^{-} K^{+} K^{-}\right)-m\left(\mu^{+} \mu^{-}\right)$) bins and the $J / \psi \phi K^{+}$mass distributions are fitted to extract the B signal yield in each of these $\Delta m$ bins. The obtained $\Delta m$ spectrum is fitted interpreting the observed structures as the $J / \psi \phi$ resonances with S-wave relativistic Breit-Wigner line-shapes over a three-body phase space non-resonant component (Fig. 3). Taking into account the known $J / \psi$ mass, CMS observed a narrow structure in the $J / \psi \phi$ system with mass $m_{1}=$ $4148.2 \pm 2.0$ (stat.) \pm 5.2 (syst.) $\mathrm{MeV}$ with a significance greater than $5 \sigma$. This observation is consistent with the previous narrow structure near $J \psi \phi$ threshold reported by CDF. CMS collaboration, also saw evidence for a second structure where the mass is measured as $m_{2}=4316.7 \pm$ 3.0 (stat.) \pm 10.0 (syst.) $\mathrm{MeV}$

\subsection{Production of $X(3872)$ via decays to $J / \psi \pi^{+} \pi^{-}$}

$\mathrm{X}(3872)$ has been observed in several decay channels, such as $J / \psi \pi^{+} \pi^{-}, D^{*} \overline{D^{0}}, \gamma J / \psi, \gamma \psi(2 S)$, and $\omega J / \psi$ since its discovery in 2003 [1]. Studies showed that X(3872) states are produced both directly (prompt) and through the decays of $\mathrm{B}$ hadrons (non-prompt). The observed decay channels suggest that it might be $D^{*} \overline{D^{0}}$ molecule or a tetra-quark state [11]. The angular analysis in the $J / \psi \pi^{+} \pi^{-}$decay channel favors the quantum number $J^{P C}$ $=1^{++}$or $2^{-+}[12,13]$. The first study based on the data collected at $\mathrm{LHC}$ is performed by the $\mathrm{LHCb}$ collaboration using $34.7 \mathrm{pb}^{-1}$ pp collision data collected during 2010 . By selecting candidates in the $\mathrm{X}(3872) \rightarrow J / \psi \pi^{+} \pi^{-}$decay mode the LHCb experiment measured the $\sigma(p p \rightarrow$ $X(3872)+$ anything $) \times \mathcal{B}\left(X(3872) \rightarrow J / 4 \pi^{+} \pi^{-}\right)$as $5.4 \pm$ 1.3 (stat.) \pm 0.8 (syst.) $\mathrm{nb}$ for the $\mathrm{X}(3872)$ mesons with $\eta$ within the 2.4-4.5 rapidity range and the $p_{T}$ is within the 5-20 GeV range [14]. The CMS experiment also studied the X(3872) in the $\mathrm{X}(3872) \rightarrow J / \psi\left(\rightarrow \mu^{+} \mu^{-}\right) \pi^{+} \pi^{-}$decay channel using $4.8 \mathrm{fb}^{-1}$ pp collision data collected during 2011 [15]. The measurements are performed for the $\mathrm{X}(3872)$ candidates with a rapidity $|\eta|<1.2$ and with 10 $50 \mathrm{GeV}$ transverse momentum range. The CMS collaboration measured

- the ratio of the cross section times branching fractions for the $\mathrm{X}(3872)$ and $\psi(2 S)$. The ratio with and without acceptance corrections for the exclusive $p_{T}$ bins are shown in Fig 4. The exclusive ratio for $10-50 \mathrm{GeV}$ $p_{T}$ region without acceptance correction is measured as $\mathrm{R}=0.0662 \pm 0.0038$ (stat) \pm 0.0064 (syst).

- the fraction of $\mathrm{X}(3872)$ originating from $\mathrm{B}$ hadron decay $(0.259 \pm 0.029$ (stat.) \pm 0.016 (syst.)) .

- the cross section times the branching fractions for the prompt $\mathrm{X}(3872)$ production using the previous CMS measurements for the prompt $\psi(2 S)$ production cross section [16]. The measured value of $\sigma^{\text {prompt }}(p p \rightarrow$ $X(3872)+$ anything $) \times \mathcal{B}\left(X(3872) \rightarrow J / \psi \pi^{+} \pi^{-}\right)$is 1.03 $\pm 0.11 \pm 0.15 \mathrm{nb}$ for the $\mathrm{X}(3872)$ candidates with $10-30$ $\mathrm{GeV} p_{T}$ range.

- the dipion mass spectrum for $\mathrm{X}(3872) \rightarrow J / \psi \pi^{+} \pi^{-}$decay mode (Fig. 5). The comparison of the measured spectrum with simulations where the samples are produced with and without intermediate $\rho^{0}$ decay shows that studied decay mode favors the presence of and intermediate $\rho^{0}$ state.

\subsection{Observation of $\chi_{b}(3 P)$ state}

The $b \bar{b}$ quakonium system includes S-wave $\Upsilon$ and the $\mathrm{P}$ wave $\chi_{b}$ states where the P-wave states are composed of closely spaced triplet $\left(\chi_{b 0}, \chi_{b 1}, \chi_{b 2}\right)$ with $\mathrm{J}=0,1,2$ spin states. Before LHC became operational previous experiments has observed the $\chi_{b}(1 \mathrm{P})$ and $\chi_{b}(2 \mathrm{P})$ states but not the $\chi_{b 0}(3 \mathrm{P})$ state. The ATLAS experiment studied the $\chi_{b}$ quarkonium states by reconstructing the radiative decay modes $\chi_{b}(n P) \rightarrow \Upsilon(1 S, 2 S) \gamma$ where $\Upsilon(1 S, 2 S) \rightarrow \mu^{+} \mu^{-}$. ATLAS experiment used both the converted (conversion 

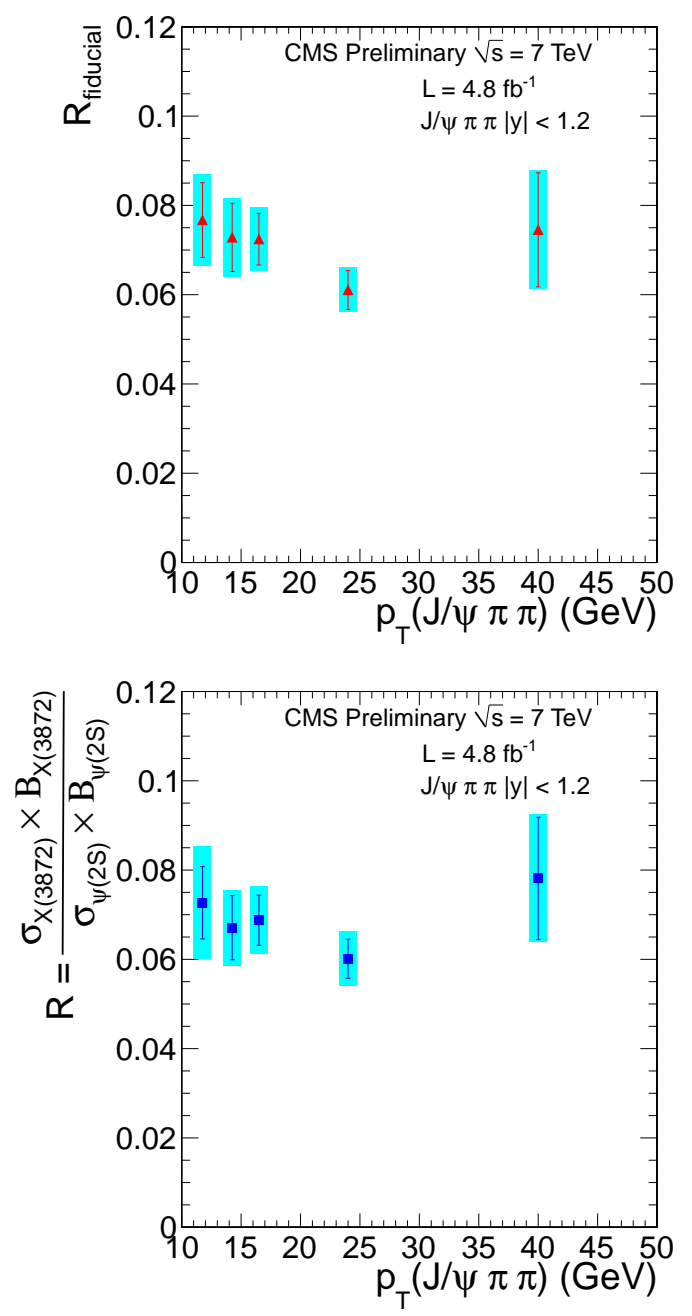

Figure 4. $\sigma \times \mathcal{B}$ ratios of the $\mathrm{X}(3872)$ and $\psi(2 S)$, without (top) and with (bottom) acceptance corrections for the muon and the pion pairs. The statistical errors are indicated by the error bars whereas the bands represent the total uncertainty.

through $e^{+} e^{-}$) and unconverted (direct calorimetric measurement) photons in this study. The invariant mass difference $\Delta m\left(\equiv m\left(\mu^{+} \mu^{-} \gamma\right)-m\left(m u^{+} \mu^{-}\right)\right)$is used by the ATLAS collaboration in order to minimize the mass resolution effect of $\Upsilon \rightarrow \mu^{+} \mu^{-}$reconstruction and the final study is performed on the variable $\tilde{m}_{k}=\Delta m+m_{\Upsilon(k S)}$ where the $m_{\Upsilon(k S)}$ is the world average masses of the $\Upsilon(k S)$ states. The $\tilde{m}_{k}$ distribution for the converted (bottom) and unconverted (top) photons are given in Fig 6. Additional to the expected $\chi_{b}(1 P, 2 P) \rightarrow \Upsilon(1 S, 2 S) \gamma$ peaks a third structure is observed in the $\tilde{m}_{k}$ distributions and interpreted as the first time observed radiative decays of the $\chi_{b}(3 P)$ states. The mass of the $\chi_{b}(3 P)$ states is measured as $10.530 \pm$ 0.005 (stat.) \pm 0.009 (syst.) $\mathrm{GeV}$ [17].

First confirmation of the $\chi_{b}(3 P)$ states came from D0 collaboration with a mass measurement $m_{\chi_{b}(3 P)}=10.551$ \pm 0.014 (stat.) \pm 0.017 (sys) $\mathrm{GeV}$ [18]. Later on $\mathrm{LHCb}$ also reported the observation of the $\chi_{b}(3 P)$ states with a significance greater than $12 \sigma$ using $0.9 \mathrm{fb}^{-1}$ of LHC data. The mass, signal yield, and the width measurements of the

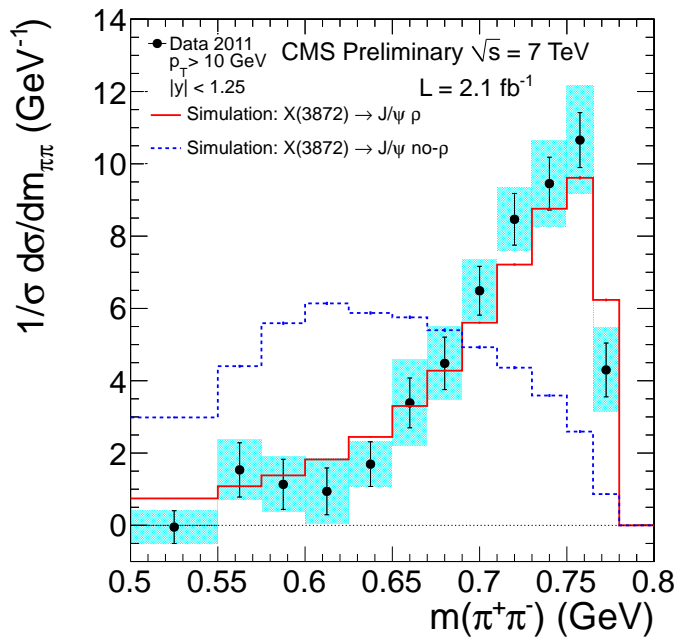

Figure 5. Pion-pair invariant mass spectrum for $X(3872) \rightarrow$ $J / \psi \pi^{+} \pi^{-}$decays corrected for acceptance and efficiency. The bands represent the total uncertainty. The results are compared to model calculations with and without an intermediate $\rho^{0}$ decay.
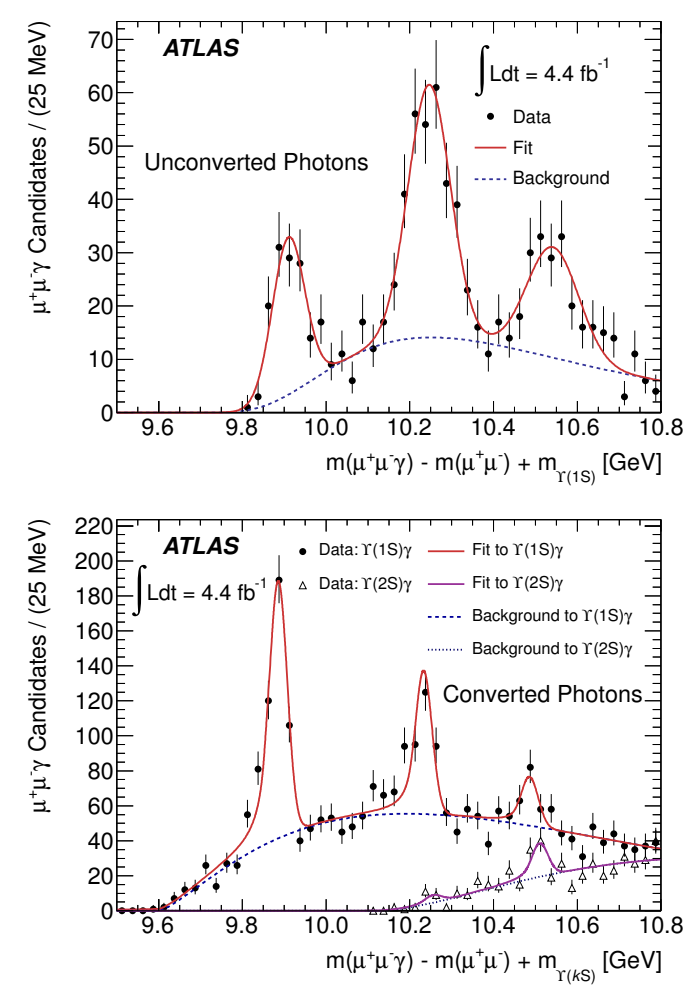

Figure 6. The mass distribution of $\chi_{B} \rightarrow \Upsilon(1 S) \gamma$ candidates for unconverted photons (top) and for the $\chi_{B} \rightarrow \Upsilon(k S) \gamma(\mathrm{k}=1,2)$ candidates formed using converted photons (bottom). The solid lines represent the total fit and the dashed lines represent the background components. 
Table 1. The yields, masses and widths of the $\chi_{b}$ states extracted from the fit of the $m\left(\mu^{+} \mu^{-} \gamma\right)-m\left(\mu^{+} \mu^{-}\right)$distribution. The uncertainties are statistical only.

\begin{tabular}{cccc}
\hline & Yield & Mass $\left(\mathrm{MeV} / \mathrm{c}^{2}\right)$ & Width $\left(\mathrm{MeV} / \mathrm{c}^{2}\right)$ \\
\hline$\chi_{b}(1 \mathrm{P})$ & $625 \pm 50$ & $9901 \pm 2$ & $25 \pm 2$ \\
$\chi_{b}(2 \mathrm{P})$ & $323 \pm 32$ & $10266 \pm 6$ & $57 \pm 6$ \\
$\chi_{b}(3 \mathrm{P})$ & $196 \pm 19$ & $10535 \pm 10$ & $80 \pm 10$ \\
\hline
\end{tabular}

LHCb experiments for the observed three $\chi_{b}(3 P)$ states $\left(\chi_{b}(1 P), \chi_{b}(2 P), \chi_{b}(3 P)\right)$ are summarized in Table 1.

\section{B-hadrons and B-baryons}

\section{1 $\Lambda_{b}^{0}$ lifetime and mass measurement}

With a mass about $5620 \mathrm{MeV}$ the $\Lambda_{b}^{0}$ baryon is the lightest baryon containing a b quark. Although it is measured several times in the past, the lifetime measurements suffers from the large experimental uncertainties. Since LHC provides large samples, both ATLAS and CMS experiments measured the $\Lambda_{b}^{0}$ lifetime in the decay channel $\Lambda_{b}^{0} \rightarrow J / \psi\left(\rightarrow \mu^{+} \mu^{-}\right) \Lambda^{0}\left(\rightarrow p \pi^{-}\right)$.

The ATLAS collaboration used $4.9 \mathrm{fb}^{-1} \mathrm{pp}$ collision data collected during 2011 at the LHC at $7 \mathrm{TeV}$ center of mass energy and reconstructed $2200 \Lambda_{b}^{0}$ and $\bar{\Lambda}_{b}^{0}$ baryon candidates. The candidates are fitted by applying a simultaneous mass and decay time maximum fit to extract the following lifetime and mass measurements [20];

$$
\begin{aligned}
& \tau_{\Lambda_{b}}=1.449 \pm 0.036 \text { (stat.) } \pm 0.017 \text { (syst.) ps } \\
& m_{\Lambda_{b}}=5619.7 \pm 0.7 \text { (stat.) } \pm 1.1 \text { (syst.) } \mathrm{MeV}
\end{aligned}
$$

The CMS experiment determined the $\Lambda_{b}^{0}$ lifetime using $5 \mathrm{fb}^{-1}$ data collected during 2011 by a applying twodimensional unbinned extended maximum likelihood fit to the measured lifetime of the selected $\Lambda_{b}^{0}$ candidates (1012 \pm 40 ) [21]. The mass (left) and the lifetime (right) projections of the two-dimensional lifetime fit for $\Lambda_{b}^{0}$ are shown in Fig. 7. CMS determined the $\Lambda_{b}^{0}$ lifetime as $1.503 \pm$ 0.052 (stat.) \pm 0.031 (sys.) ps which is consistent with the world average value of $1.425 \pm 0.032 \mathrm{ps}$ [22]

\subsection{Observation of the excited $\Lambda_{b}^{0}$ baryons}

Existence of the orbitally excited $\Lambda_{b}^{0}$ states are predicted by the quark model and studied by the LHCb experiment using $1.0 \mathrm{fb}^{-1} \mathrm{pp}$ collision data collected by LHCb detector. The two excited states with the quantum numbers $J^{P}$ $=1 / 2^{-}$and $3 / 2^{-}$and expected to decay to $\Lambda_{b}^{0} \pi^{+} \pi^{-}$or $\Lambda_{b}^{0} \gamma$. The $\Lambda_{b}^{0}$ candidates are reconstructed by following decay channel where $\Lambda_{b}^{0} \rightarrow \Lambda_{c}^{+}\left(\rightarrow p K^{-} \pi^{+}\right) \pi^{-}$. The selected $\Lambda_{b}^{0}$ candidates are combined with the two tracks under the pion mass assumption and used for searching the excited $\Lambda_{b}^{0}$ states. The invariant mass of the $\Lambda_{b}^{0} \pi^{+} \pi^{-}$is shown in Fig 8. The observed two narrow structures with masses around 5912 and $5920 \mathrm{MeV} / \mathrm{c}^{2}$ are interpreted as the orbitally excited $\Lambda_{b}^{0}$ states $\left(\Lambda_{b}^{* 0}(5912)\right.$ and $\left.\Lambda_{b}^{* 0}(5920)\right)$. Those two structures are observed with signal significances 4.9 and $10.2 \sigma$, respectively. The precise masses
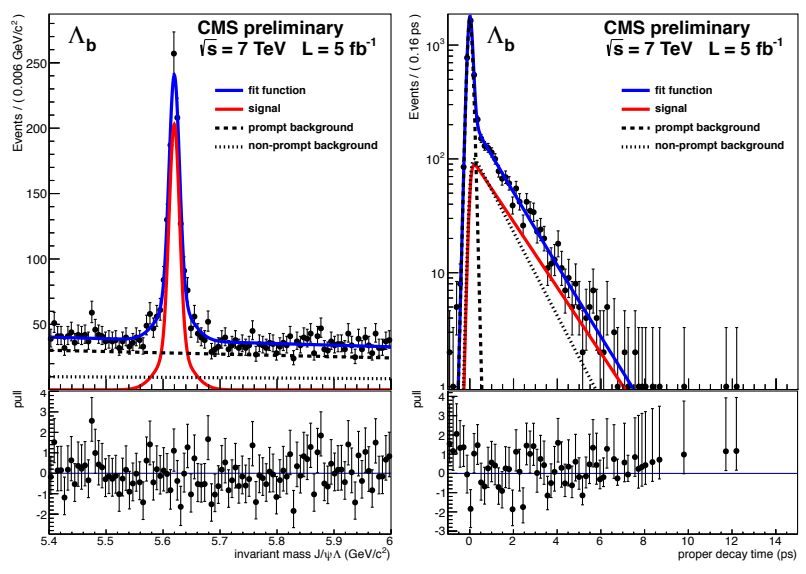

Figure 7. Result for two-dimensional lifetime fit for $\Lambda_{b}$. Shown are the projections to the mass domain (left) and the time domain (right). The curves correspond to the full fit curve and the three components signal, prompt background and non-prompt background.

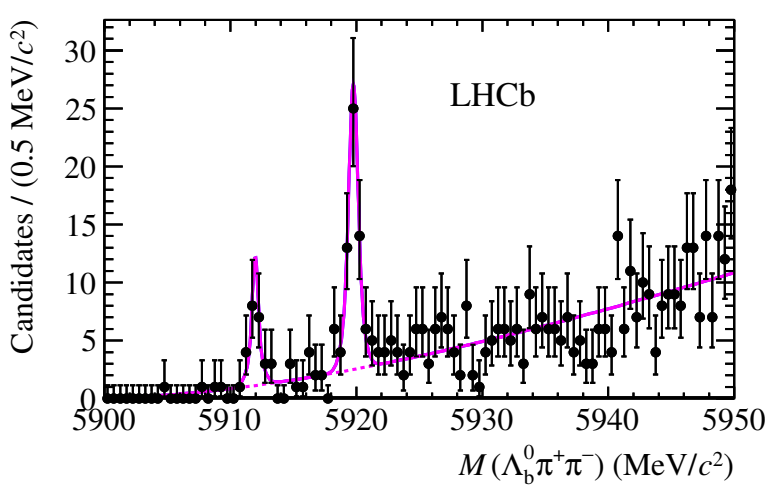

Figure 8. Invariant mass spectrum of $\Lambda_{b} \pi^{+} \pi^{-}$. The points with error bars are the data, the solid line is the fit result, the dashed line is the background contribution.

for the observed excited states are;

$$
\begin{aligned}
& M_{\Lambda_{b}^{\star 0}(5912)}=5911.95 \pm 0.12 \pm 0.03 \pm 0.66 \mathrm{MeV} / \mathrm{c}^{2} \\
& M_{\Lambda_{b}^{\circ 0}(5920)}=5919.76 \pm 0.07 \pm 0.02 \pm 0.66 \mathrm{MeV} / \mathrm{c}^{2}
\end{aligned}
$$

where the first uncertainty is statistical, the second one is the systematic, and the last uncertainty is due to the precision of the known $\Lambda_{b}^{0}$ mass.

\subsection{Observation of $\Xi_{b}^{*}$ baryon}

The first discovery of CMS was the observation of a new $\mathrm{b}$ baryon via its strong decay into the $\Xi_{b}^{-} \pi^{+}$and its charge conjugates [24]. The observation is done through the analysis of $5.3 \mathrm{fb}^{-1} \mathrm{pp}$ collision data collected with CMS detector. The the $\Xi_{b}^{-}$baryon is reconstructed by the decay chain $\Xi_{b}^{-} \rightarrow J / \psi\left(\rightarrow \mu^{+} \mu^{-}\right) \Xi^{-}\left(\rightarrow \Lambda^{0} \pi^{-}\right)$where $\Lambda^{0}$ decays to a proton and a $\pi^{-}$. In the performed analysis a peak is observed in the $\mathrm{Q}\left(\equiv M\left(J / \psi \Xi^{-} \pi^{+}\right)-M\left(J / \psi \Xi^{-}\right)-M\left(\pi^{+}\right)\right)$ distribution with a significance greater than $5 \sigma$. The mass of the observed peak is $14.84 \pm 0.74$ (stat.) \pm 0.28 (sys) 
$\mathrm{MeV}$. Taking into account the charged pion and the $\Xi_{b}^{-}$ masses, the mass of the new b baryon is $5945.0 \pm 0.7$ (stat.) \pm 0.3 (sys.) \pm 2.7 (PDG) $\mathrm{MeV}$. The last contribution is from the current accuracy of the measured $\Xi_{b}^{-}$mass. The width of the observed peak was not able to be measured with good precision due to the statistical limitations however the measured value is consistent with the lattice QCD calculations [25]. The extracted mass and the observed decay mode indicates that the observed new baryon most likely is the $\Xi_{b}^{* 0}$ with $J^{P}=3 / 2^{+}$.

\section{4 $B_{S}$ lifetime difference measurement}

Mixing between the two flavor eigenstates of the $B_{s}$ meson results mass eigenstates which are expected to have measurable mass and the decay difference. In the SM, the $\mathrm{CP}$-violating mixing phase is predicted to be small and the two mass eigenstates are approximately equal to $\mathrm{CP}$ eigenstates. By using $5.0 \mathrm{fb}^{-1} \mathrm{pp}$ collision data, CMS performed an angular and proper decay time analysis to measure the $\Delta \Gamma_{s}$ and the polarization amplitudes with assuming the mixing phase $\left(\phi_{s}\right)$ is equal to zero. The lifetime difference in the $B_{s} \rightarrow J / \psi\left(\mu^{+} \mu^{-}\right) \phi\left(K^{+} K^{-}\right)$decay is measured by performing a flavor untagged analysis. A total $14456 B_{s}$ candidates are reconstructed and a five dimensional unbinned maximum likelihood fit is applied to the data to measure the decay width difference between the two $B_{s}$ mass eigenstates. The decay with difference is measured as $\Delta \Gamma_{s}=0.048 \pm 0.024$ (stat.) \pm 0.003 (sys.) $p s^{-1}[26]$.

\section{Summary}

Spectroscopy and the properties of the B-hadrons and the heavy quarkonium sector are presented from the perspective of ATLAS, CMS and the LHCb experiments. The experiments obtained precise results with smaller systematic errors thanks to the large data sample collected during 2011 at a new energy regime. The $\Lambda_{b}^{0}$ lifetime measurements by ATLAS and CMS, the properties of X(3872) by CMS are summarized and new observations such as $\chi_{b}(3 P)$ state by ATLAS, $\Xi_{b}^{*}$ baryon by CMS, orbitally excited $\Lambda_{b}^{0}$ baryons by $\mathrm{LHCb}$, and the structure in the $J / \psi \phi$ mass spectrum are reported.

\section{References}

[1] Belle Collaboration, Phys. Rev. Lett. 91, 262001 (2003)
[2] Belle Collaboration, Phys. Rev. Lett. 94, 182002 (2005)

[3] BABAR Collaboration, Phys. Rev. Lett. 101, 082001 (2008)

[4] BABAR Collaboration, Phys. Rev. Lett. 95, 142001 (2005)

[5] CLEO Collaboration, Phys. Rev. D 74, 091104(R) (2006)

[6] CDF Collaboration, Phys. Rev. Lett. 102, 242002 (2009)

[7] CDF Collaboration, arXiv:1101.6058v1 (2011)

[8] LHCb Collaboration, Phys. Rev. D 85, 091103(R) (2012)

[9] Particle Data Group Collaboration, J. Phys. G 37 075021 (2010)

[10] CMS Colloboration, Public Physics Results, (2012) twiki.cern.ch/twiki/bin/viewauth/CMS/PhysicsResultsBPH11026

[11] G. Bauer, Int. J. Mod. Phys. A, 20, 3765 (2005)

[12] Belle Collaboration, Phys. Rev. D 84, 052004 (2011)

[13] CDF Collaboration, Phys. Rev. Lett. 98, 132002 (2007)

[14] LHCb Collaboration, The Eur. Phys. J. C. 72, 1972 (2012)

[15] CMS Colloboration, Public Physics Results, (2012) twiki.cern.ch/twiki/bin/viewauth/CMS/PhysicsResultsBPH11011

[16] CMS Collaboration, JHEP 02, 011 (2012)

[17] ATLAS Collaboration, Phys. Rev. Lett. 108, 152001 (2012)

[18] D0 Collaboration, arXiv:1203.6034,(2012)

[19] LHCb Collaboration, arXiv:1203.6034, LHCbCONF-2012-020, (2012)

[20] ATLAS Collaboration, arXiv:1207.2284v2, (2012)

[21] CMS Colloboration, Public Physics Results, (2012) twiki.cern.ch/twiki/bin/viewauth/CMS/PhysicsResultsBPH11013

[22] Beringer et al. (PDG), Phys. Rev. D 86, 010001 (2012)

[23] LHCb Collaboration, Phys. Rev. Lett. 109, 172003 (2012)

[24] CMS Colloboration, Phys. Rev. Lett. 108, 252002 (2012)

[25] W. Detmold, C.-J. D. Lin, and S. Meinel, arXiv:1203.3378 (2012).

[26] CMS Colloboration, CMS-PAS-BPH-11-006, (2012) 